\title{
ON THE PRIMITIVITY OF HOPF ALGEBRAS OVER A FIELD WITH PRIME CHARACTERISTIC
}

\author{
EDWARD HALPERN
}

We recall that an $H$-space consists of a topological space $T$ with a base point $e \in T$ and a (continuous) map $\nabla: T \times T \rightarrow T$ such that $\nabla i \simeq I$ and $\nabla j \simeq I$, where $i$ and $j$ are defined by $i(t)=(t, e)$ and $j(t)$ $=(e, t), I$ is the identity map of $T$, and " $\simeq$ " means "homotopic relative to $e$." The multiplication $\nabla$ is homotopy-associative if

$$
\nabla(\nabla \times I) \simeq \nabla(I \times \nabla)
$$

it is homotopy-commutative if

$$
\rho \nabla \simeq \nabla
$$

where $\rho$ is defined by $\rho(s, t)=(t, s),(s, t \in T)$. We shall assume throughout that $T$ is arcwise connected.

Let $H$ be an associative and anticommutative graded $K$-algebra with unit 1 , where $K$ is a field. We assume throughout that $H^{i}=0$ if $i<0$, and $H^{0}=K \cdot 1$. Let $H^{+}$denote the submodule spanned by the elements of positive degree. $H$ is a Hopf algebra over $K$ if there is an algebra homomorphism $\Delta: H \rightarrow H \otimes H$ (regarding $H \otimes H$ as a graded $K$-algebra in the usual way) such that

$$
\Delta^{\prime \prime}(x)=\Delta(x)-\Delta^{\prime}(x) \in H^{+} \otimes H^{+}, \quad x \in H,
$$

where $\Delta^{\prime}: H \rightarrow H \otimes H$ is defined by

$$
\Delta^{\prime}(1)=1 \otimes 1, \quad \Delta^{\prime}(x)=x \otimes 1+1 \otimes x, \quad x \in H^{+} .
$$

We shall refer to $\Delta$ as the coproduct.

The coproduct is associative if

$$
(\Delta \otimes I) \Delta=(I \otimes \Delta) \Delta
$$

where $I$ is the identity map of $H$; it is anticommutative if

$$
\theta \Delta=\Delta,
$$

where $\theta$ is defined by

$$
\theta(x \otimes y)=(-1)^{i j} y \otimes x, \quad x \in H^{i}, y \in H^{i} .
$$

By a Hopf subalgebra we mean a graded subalgebra $G$ such that $\Delta(G) \subset G \otimes G$.

Presented to the Society, January 22, 1959; received by the editors April 16, 1959. 
It is well-known that the cohomology algebra $H^{*}(T, K)$, where $T$ is an $H$-space and $K$ is a field, is a Hopf algebra with coproduct $\Delta=\nabla^{*}$ (assuming the usual identification given by the Künneth formula). Comparing (1.1) and (1.3), evidently homotopy-associativity of $\nabla$ implies associativity of $\Delta$. It is known that $\theta=\rho^{*}$; hence, comparing (1.2) and (1.4), we see that homotopy-commutativity of $\nabla$ implies anticommutativity of $\Delta$.

Let $(H, \Delta)$ be a Hopf algebra over $K$. An element $y \in H$ is primitive if $\Delta^{\prime \prime}(y)=0$. Let $\pi \subset H$ be the subalgebra generated by the primitive elements. It is easy to see that $\pi$ is a Hopf subalgebra. If $\pi=H$ then we call $H$ a primitive Hopf algebra. The following theorem was proved by the author [3, Theorem 2.10], and independently by J. C. Moore [5].

(1.5) If $H$ is a Hopf algebra over a field of characteristic zero and the coproduct is associative and anticommutative then $H$ is primitive.

A simple algebraic example shows that (1.5) is not true in general if the field has prime characteristic. The following theorem is due to H. Samelson [6] and J. Leray [4]:

(1.6) Let $H$ be a Hopf algebra over a field and let the coproduct be associative. If $H$ is an exterior algebra generated by odd degree elements then it is primitive.

The purpose of this paper is to establish primitivity of $H^{*}\left(T, Z_{p}\right)$ for some $H$-spaces $T$, where $Z_{p}$ is the ring integers modulo a prime $p$. We shall make use of properties of the Steenrod cohomology operations [7] which we denote by

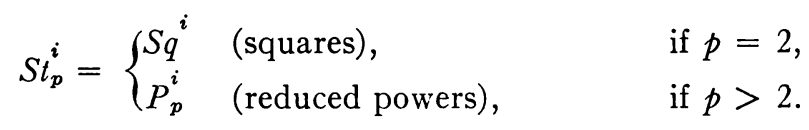

Let $T$ be an $H$-space and suppose $H^{*}\left(T, Z_{p}\right)$ is a polynomial ring $Z_{p}[X]$ where $X \subset H^{*}\left(T, Z_{p}\right)$ and consists of even degree elements if $p \neq 2$. The operations $S t_{p}^{i}$ are said to split on $X$ if for all $i \geqq 0$ and $x \in X, S t_{p}^{i}(x)$ is in the subalgebra generated by $x$.

THEOREM 1. Let $T$ be an arcwise connected $H$-space with homotopyassociative and homotopy-commutative multiplication. If $H^{*}\left(T, Z_{p}\right)$ $=Z_{p}[X]$ and the Steenrod cohomology operations split on $X$ then $H^{*}\left(T, Z_{p}\right)$ is primitive.

We remark that it then follows on using a Künneth formula that $H^{*}(T, K)$ is primitive if $K$ is a field of characteristic $p$.

As an application of Theorem 1 we shall prove the following theorem. For a fixed prime $p$, a topological space $E$ is p-elementary if 
$H^{*}\left(E, Z_{p}\right) \cong Z_{p}$ or $H^{*}\left(E, Z_{p}\right)=Z_{p}[x]$. As examples we cite: The real projective plane $(p \neq 2)$, the loop spaces $\Omega\left(S^{2 n+1}\right)$ and complex projective space of infinite dimensions. On the other hand the loop spaces $\Omega\left(S^{2 n}\right)$ are not $p$-elementary for any $p$.

Theorem 2. Let $p$ be a fixed prime and $E=E_{1} \times \cdots \times E_{n}$, where the $E_{i}$ are p-elementary spaces. Let $T$ be an arcwise connected $H$-space with homotopy-associative and homotopy-commutative multiplication. If there is a map $f: T \rightarrow E$ or $f: E \rightarrow T$ which induces an isomorphism of the cohomology algebras with coefficients in $Z_{p}$, then $H^{*}\left(T, Z_{p}\right)$ is primitive.

Proof. Using the Künneth formula and $f^{*}$ we may represent

$$
H^{*}\left(T, Z_{p}\right)=Z_{p}\left[x_{1}, x_{2}, \cdots, x_{n}\right]
$$

where $x_{i}$ generates $H^{*}\left(E_{i}, Z_{p}\right)$ (we may ignore the trivial factors). In view of the Cartan tensor product formula (see [2, Exposé 16, bis 1]) the $S t_{p}^{i}$ split on $H^{*}\left(E_{1} \times \cdots \times E_{n}, Z_{p}\right)$ and hence on $\left\{x_{1}, x_{2}, \cdots, x_{n}\right\}$ since they commute with $f^{*}$. Thus the theorem follows from Theorem 1 .

Corollary. A necessary condition that an arcwise connected $H$-space $T$ with homotopy-associative and homotopy-commutative multiplication be homotopically equivalent to a cartesian product of p-elementary spaces $E_{1}, E_{2}, \cdots, E_{n}$ is that $H^{*}\left(T, Z_{p}\right)$ be primitive.

REMARK. Even if the $E_{i}$ are all $H$-spaces, the map $f$ is not required to commute with the multiplication in $T$ and the induced multiplication in $E_{1} \times E_{2} \times \cdots \times E_{n}$.

2. The main lemma. Let $H=K[X]$ be a Hopf algebra over a field $K$ of prime characteristic $p$. We note:

(2.1) If $p \neq 2$ each $x \in X$ has even degree.

(2.2) We may assume that each $x \in X \cap \pi$ is primitive.

The first is a well-known consequence of the theorem of A. Borel (see [1, Théorème 6.1]). The second follows from Theorem 2.7 in [3].

We shall assume throughout that $X$ is well-ordered in such a way that if $x$ has lower degree than $y$ then $x<y$. By a normal monomial we shall mean a product of the form $M=x_{1}^{m_{1}} x_{2}^{m_{2}} \cdots x_{t}^{m_{t}}$, where the $x_{i} \in X$ and $x_{i}<x_{i+1}$. We call $m_{1}+\cdots+m_{t}$ the length of $M$ and the number of positive exponents its width. If $R$ and $S$ are normal monomials their juxtaposition $R S$ (corresponding to their product as elements of $H$ ) is equal to a unique normal monomial which we denote by $\nu(R S)$. If 


$$
R=x_{1}^{r_{1}} x_{2}^{r_{2}} \cdots x_{t}^{r_{t}}, \quad S=x_{1}^{s_{1}} x_{2}^{s_{2}} \cdots x_{t}^{s_{t}}, \quad r_{i}+s_{i}=m_{i},
$$

we define

$$
[R, S]=\prod_{i=1}^{t}\left(r_{i}, s_{i}\right), \quad m_{i} ! / r_{i} ! s_{i} !=\left(r_{i}, s_{i}\right) .
$$

By induction on width one proves readily

(2.3) If $M$ is a normal monomial whose factors are primitive then

$$
\Delta(M)=\sum_{\nu(R S)=M}[R, S] R \otimes S,
$$

where the summation extends over distinct pairs of normal monomials $R, S$.

Let $z \in X$ be such that $\Delta^{\prime \prime}(z) \in \pi \otimes \pi$. Then we may write (uniquely)

$$
\Delta^{\prime \prime}(z)=\sum a(M, N) M \otimes N, \quad a(M, N) \in K,
$$

where the summation extends over finitely many distinct pairs of normal monomials $M, N$ in primitive elements of $X$ and the degree of $M N$ is equal to the degree of $z$. The proof of Theorem 1 will depend on the

MaIN Lemma. Let $H$ be a Hopf algebra with an associative and anticommutative coproduct $\Delta$ over a field $K$ with prime characteristic $p$. Let $H=K[X]$, where $X \subset H$, and let $z \in X$ be such that $\Delta^{\prime \prime}(z) \in \pi \otimes \pi$. Then there is an element $v \in H$ with the same degree as $z$ such that $z-v \in \pi$ and

$$
\Delta^{\prime \prime}(v)=\sum \sum a\left(x^{m}, x^{n}\right) x^{m} \otimes x^{n}, \quad a\left(x^{m}, x^{n}\right) \in K
$$

where the outer summation is over (primitive) $x \in X$ and the inner summation is over (positive) $m$ and $n$ with $m+n a$ power of $p$.

We shall first prove some subsidiary lemmas. It will be convenient to extend the definition of $a(M, N)$ in (2.4) as follows: If $Q, R, S, T$. are normal monomials in primitive elements of $X$ then

$$
a(Q R, S T)=a(\nu(Q R), \nu(S T)) .
$$

Lemma 2.1. If $R, S, T$ are normal monomials with $R \neq 1$ and $T \neq 1$ then

$$
a(R S, T)[R, S]=a(R, S T)[S, T] .
$$

Proof. Since the coproduct is associative we may equate the coefficients of $R \otimes S \otimes T$ in $(\Delta \otimes I) \Delta(z)$ and $(I \otimes \Delta) \Delta(z)$. Since $R \neq 1$ and $T \neq 1$, it is readily seen $(\Delta \otimes I) \Delta^{\prime}(z)$ and $(I \otimes \Delta) \Delta^{\prime}(z)$ contribute nothing to these coefficients. We have 


$$
(\Delta \otimes I) \Delta^{\prime \prime}(z)=\sum a(M, N) \Delta(M) \otimes N,
$$$$
(I \otimes \Delta) \Delta^{\prime \prime}(z)=\sum a(M, N) M \otimes \Delta(N) .
$$

Now using (2.3) it follows that the coefficients of $R \otimes S \otimes T$ in (2.6) and $(2.6)^{\prime}$, respectively, are

$$
\begin{aligned}
& a(\nu(R S), T)[R, S]=a(R S, T)[R, S], \\
& a(R, \nu(S T))[S, T]=a(R, S T)[S, T],
\end{aligned}
$$

and the lemma is proved.

LEMMA 2.2. If $\nu(M N)$ is of the form $w^{k} Q$, where $k \geqq 1$ is the multiplicity of $w$ and $Q \neq 1$ is normal then

$$
a(M, N)=[M, N] a\left(w^{k}, Q\right) .
$$

Proof. It suffices to consider $M$ and $N$ as normal monomials of the form $x^{m} R$ and $y^{n} S$ respectively, where $m$ and $n$ are the corresponding (positive) multiplicities of $x$ and $y$ and $R$ and $S$ are normal. Note that if $R=1$ and $S=1$ the lemma follows at once from anticommutativity of $\Delta$. Assume that not both $R=1$ and $S=1$; we consider 3 cases:

(i) $x<y$. If $R=1$ the lemma is trivial. If $R \neq 1$ then $a\left(x^{m} R, N\right)=[R, N] a\left(x^{m}, R N\right)=[R, N] a\left(x^{m}, Q\right)=[M, N] a\left(x^{m}, Q\right)$.

(ii) $x=y$. If $R=1$ then

$$
a\left(x^{m}, x^{n} S\right)=\left[x^{m}, x^{n}\right] a\left(x^{m+n}, Q\right)=\left[x^{m}, N\right] a\left(x^{m+n}, Q\right) .
$$

If $R \neq 1$ then

$$
\begin{aligned}
a\left(x^{m} R, x^{n} S\right) & =[R, N] a\left(x^{m}, R x^{n} S\right) \\
& =[R, N] a\left(x^{m}, x^{n} R S\right) \\
& =[R, N]\left[x^{m}, x^{n}\right] a\left(x^{m+n}, R S\right) \\
& =[R, N]\left[x^{m}, x^{n}\right] a\left(x^{m+n}, Q\right) \\
& =\left[x^{m} R, N\right] a\left(x^{m+n}, Q\right) .
\end{aligned}
$$

(iii) $x>y$. Using case (i) we may write

$$
a(N, M)=[N, M] a\left(y^{k}, Q\right) .
$$

Note that $a(M, N)=a(N, M)$ by anticommutativity of $\Delta$.

Lemma 2.3. If $x \in X, m+n=r+s$, and

$$
(s-n, n) \not \equiv 0 \quad(\bmod p)
$$$$
s \geqq n,
$$

then 


$$
(r, s) a\left(x^{m}, x^{n}\right)=(m, n) a\left(x^{r}, x^{s}\right) .
$$

Proof. By Lemma 2.1,

$$
(r, m-r) a\left(x^{m}, x^{n}\right)=(s-n, n) a\left(x^{r}, x^{s}\right) .
$$

If we multiply by $(m, n)$ and use the identity

$$
(m, n)(r, m-r)=(r, s)(s-n, n),
$$

we get

$$
(r, s)(s-n, n) a\left(x^{m}, y^{n}\right)=(m, n)(s-n, n) a\left(x^{r}, x^{s}\right) .
$$

In view of $(2.7)$ we may divide out $(s-n, n)$.

Lemma 2.4. If $x \in X$ and $m+n=q p^{i}$, where $q>1$ and $q \neq \equiv 0(\bmod p)$ then

(2.8) $a\left(x^{m}, x^{n}\right)=0$ if $p^{i}$ does not divide $m$ and $n$,

(2.9) $a\left(x^{r p^{i}}, x^{s p^{i}}\right)=(r, s) a\left(x^{(q-1) p^{i}}, x^{p^{i}}\right) / q \quad$ if $s \not \equiv 0(\bmod p)$.

ProOF OF (2.8). Suppose $n<(q-1) p^{i}$. Since $n$ is not divisible by $p^{i}$,

$$
\left((q-1) p^{i}-n, n\right) \not \equiv 0
$$

hence by Lemma 2.3,

$$
q a\left(x^{m}, x^{n}\right)=(m, n) a\left(x^{p^{i}}, x^{(q-1) p^{i}}\right) .
$$

Since $m$ and $n$ are not divisible by $p^{i},(m, n) \equiv 0(\bmod p)$ and $(2.8)$ follows. If $n>(q-1) p^{i}$ then $m<(q-1) p^{i}$ and hence $a\left(x^{n}, x^{m}\right)=0$. By anticommutativity of $\Delta,(2.8)$ follows.

Proof of (2.9). Note that

$$
\left(s p^{i}-p^{i}, p^{i}\right)=\left((s-1) p^{i}, p^{i}\right) \equiv(s-1,1)=s \not \equiv 0, \quad(\bmod p) .
$$

Therefore (2.9) is obtained on applying Lemma 2.3.

Proof of the MaIn Lemma. Let $V$ be a normal monomial composed of primitive factors and of the same degree as $z$. We consider two types of $V$ :

(i) $V$ has width greater than 1 . Then we may write $V=x^{r} S$, where $x$ is the first factor of $V$ and its multiplicity is $r \geqq 1$, and $S \neq 1$. Put $a(V)=a\left(x^{r}, S\right)$. Then using (2.3) and Lemma 2.2, we may write

$$
\begin{aligned}
\Delta^{\prime \prime}(a(V) V) & =\sum_{V=\nu(M N) ; M \neq 1, N \neq 1} a(V)[M, N] M \otimes N \\
& =\sum_{V=\nu(M N) ; M \neq 1, N \neq 1} a(M, N) M \otimes N .
\end{aligned}
$$

It follows that if $M \neq 1, N \neq 1$, and $\nu(M N)=V$ then $M \otimes N$ has zero coefficient in $\Delta^{\prime \prime}(z-a(V) V)$. 
(ii) $V=x^{q p^{i}}$, where $q>1$ and $q \not \equiv 0(\bmod p)$. Put

$$
a(V)=a\left(x^{(q-1) p^{i}}, x^{p^{i}}\right) / q .
$$

Using (2.3) we may write

$$
\Delta^{\prime \prime}(a(V) V)=\sum_{r, s>0 ; r+s=q} a(V)(r, s) x^{r p^{i}} \otimes x^{s p i} .
$$

Applying (2.9) to the terms for which $s \not \equiv 0$ we may write

$$
\begin{aligned}
\Delta^{\prime \prime}(a(V) V)= & \sum_{s \neq 0} a\left(x^{r p i}, x^{s p^{p}}\right) x^{r p^{i}} \otimes x^{s p^{i}} \\
& +\sum_{s \equiv 0} a(V)(r, s) x^{r p i} \otimes x^{s p^{i}} .
\end{aligned}
$$

We assert that if $m+n=q p^{i}$ then $x^{m} \otimes x^{n}$ has zero coefficient in $\Delta^{\prime \prime}(z-a(V) V)$. In view of (2.8) only terms with $m$ and $n$ both divisible by $p^{i}$ can occur. In view of (2.10) only terms with

$m=r p^{i}, \quad n=s p^{i}, \quad r+s=q, \quad r>0, \quad s>0, \quad s \equiv 0(\bmod p)$

can occur. But $s \equiv 0$ and $q \not \equiv 0$ imply $r \not \equiv 0$. Thus, since $\Delta$ is anticommutative,

$$
a\left(x^{r p^{i}}, x^{s p^{i}}\right)=a\left(x^{s p^{i}}, x^{r p^{i}}\right)=0,
$$

and the assertion is proved.

Now define

$$
v=z-\sum a(V) V
$$

where the summation extends over all $V$ of types (i) and (ii). Then $v$ evidently has the properties asserted in the main lemma.

3. Proof of Theorem 1. Let $H^{*}\left(T, Z_{p}\right)=Z_{p}[X]$; assume that the elements of $X \cap \pi$ are primitive (see (2.2)). If $H^{*}\left(T, Z_{p}\right)$ is not primitive then there is an element $z \in X$ which is not in $\pi$. Moreover, if we take $z$ of lowest degree then $\Delta^{\prime \prime}(z) \in \pi \otimes \pi$ and we may write (2.4). Since $\Delta=\nabla^{*}$ is associative and anticommutative, there is an element $v \in H$ with the properties specified by the main lemma. We shall show that $v$ is primitive; this will produce a contradiction for it implies that $z \in \pi$.

We shall make use of the following properties of $S t_{p}^{4}$ :

$$
S t_{p}^{i}: H^{q}\left(T, Z_{p}\right) \rightarrow H^{q+r(p-1)}\left(T, Z_{p}\right)
$$

where $r=i$ if $p=2$ and $r=2 i$ if $p \neq 2$.

$$
S t_{p}^{i} \Delta=\Delta S t_{p}^{i}
$$


where

$$
\begin{aligned}
& S t_{p}^{i}(u \otimes w)=\sum_{i=j+k} S t_{p}^{j}(u) \otimes S t_{p}^{k}(w) . \\
& S t_{p}^{i}(u)=\left\{\begin{array}{lll}
u, & \text { if } & i=0, \\
u^{p}, & \text { if } & r=\text { degree of } u, \\
0, & \text { if } & r>\text { degree of } u,
\end{array}\right.
\end{aligned}
$$

where $r$ is as defined above.

From (3.3) and (3.4) it follows that $S t_{p}^{i}$ commutes with $\Delta^{\prime}$ and hence also with $\Delta^{\prime \prime}$ in view of (3.2). Thus

$$
S t_{p}^{i} \Delta^{\prime \prime}(v)=S t_{p}^{i} \Delta^{\prime \prime}(v-z)+\Delta^{\prime \prime} S t_{p}^{i}(z) .
$$

Now consider the expression (2.5) for $\Delta^{\prime \prime}(v)$. Let $a\left(x^{m}, x^{n}\right) x^{m} \otimes x^{n}$ be a summand such that $m d$ is maximum, where $d$ is the degree of $x$. Put

$$
a=a\left(x^{m}, x^{n}\right), \quad m+n=p^{k} .
$$

In (3.5) take $i=m j$, where $j=d$ if $p=2$, and $2 j=d$ if $p \neq 2$. We shall prove:

A. $S t_{p}^{m j}(z)=0$.

B. The coefficient of $x^{m p} \otimes x^{n}$ in $S t_{p}^{m j} \Delta^{\prime \prime}(v)$ is $a\left(x^{m}, x^{n}\right)$.

C. The coefficient of $x^{m p} \otimes x^{n}$ in $S t_{p}^{m j} \Delta^{\prime \prime}(v-z)$ is zero.

In view of (3.5) it follows from $\mathrm{A}, \mathrm{B}, \mathrm{C}$ that $a\left(x^{m}, x^{n}\right)=0$, and hence $v$ is primitive.

Proof of A. The degrees of $z$ and $S t_{p}^{m j}(z)$ are $d p^{k}$ and $d\left(p^{k}+m(p-1)\right)$, respectively. The latter is not a multiple of the former since $p^{k}>m$, $p-1$. Thus A follows from the fact that $S t_{p}^{m j}(z)$ is in the subalgebra generated by $z$.

Proof of B. We have

$$
S t_{p}^{m j}\left(a x^{m} \otimes x^{n}\right)=a x^{m p} \otimes x^{n}+\sum u_{i} \otimes w_{i}
$$

where the degrees of the $u_{i}$ are less than $m d p$. It remains to show that no other summand $b y^{r} \otimes y^{s}$ in $\Delta^{\prime \prime}(v)$ can contribute to the coefficient of $x^{m p} \otimes x^{n}$. If the degree of $y^{r}$ is less than $m d$ this is clear; if the degree of $y^{r}$ is $m d$ then, writing a similar expression to (3.6) for $S t_{p}^{m j}\left(b y^{r} \otimes y^{*}\right)$, we see that only $b y^{r p} \otimes y^{s}$ has the same bidegree as $x^{m p} \otimes x^{n}$. But if $y^{r} \otimes y^{s} \neq x^{m} \otimes x^{n}$ then $y \neq x$ or $r \neq m$, and hence $S t_{p}^{m j}\left(y^{r} \otimes y^{s}\right)$ contributes nothing to the coefficient of $x^{m p} \otimes x^{n}$.

Proof of C. Combining (2.4) and (2.5) we may write 


$$
\Delta^{\prime \prime}(v-z)=-\sum a(M, N) M \otimes N ;
$$

note that $M \otimes N$ has the property that $M N \neq y^{p i}$ for $y \in X$. Consider such a term $M \otimes N$. Let $\left(d_{1}, d_{2}\right)$ be its bidegree, and $c_{M, N}$ the coefficient of $x^{m p} \otimes x^{n}$ in $S t_{p}^{m j}(M \otimes N)$. If $d_{1}<m d$ then it is clear that $c_{M, N}=0$. If $d_{1}=m d$ then the only term in $S t_{p}^{m j}(M \otimes N)$ with the same bidegree as $x^{m p} \otimes x^{n}$ is $M^{p} \otimes N$. In view of the restriction on $M N, M^{p} \otimes N$ $\neq x^{m p} \otimes x^{n}$, and hence $c_{M, N}=0$. Finally, we complete the proof of $\mathrm{C}$ and hence of Theorem 1 by showing that if $d_{1}>m d$ then $a(M, N)=0$.

Let $M \otimes N$ be such that $d_{1}$ is maximum. In (3.5) take $i=d_{1}$ if $p=2$, and $i=d_{1} / 2$ if $p \neq 2$ (the latter is possible since if $p \neq 2, M$ has even degree by (2.1)). We assert:

$\mathrm{A}^{\prime} . S t_{p}^{i}(z)=0$.

$\mathrm{B}^{\prime}$. The coefficient of $M^{p} \otimes N$ in $S t_{p}^{4} \Delta^{\prime \prime}(v-z)$ is $-a(M, N)$.

$\mathrm{C}^{\prime}$. The coefficient of $M^{p} \otimes N$ in $S t_{p}^{i} \Delta^{\prime \prime}(v)$ is zero.

In view of (3.5), $\mathrm{A}^{\prime}, \mathrm{B}^{\prime}, \mathrm{C}^{\prime}$ imply $a(M, N)=0$. The proof of $\mathrm{C}^{\prime}$ follows immediately from $m d<d_{1}$. For if $\left(e_{1}, e_{2}\right)$ is the bidegree of a term in $S t_{p}^{i} \Delta^{\prime \prime}(v)$ then $e_{1}$ is at most $m d+d_{1}(p-1)<p d_{1}$. The proof of $\mathrm{B}^{\prime}$ is very similar to the proof of $\mathrm{B}$ and we omit the details. To prove $\mathrm{A}^{\prime}$ it suffices to show that the degree of $S t_{p}^{i}(z)$ which is $d p^{k}$ $+d_{1}(p-1)$ is not a multiple of $d p^{k}$ (the degree of $z$ ) or, equivalently, that $d_{1}(p-1)$ is not a multiple of $d p^{k}$.

Consider $a\left(x^{m}, x^{n}\right)$ again and put $m=q p^{i}$, where $q \neq 0(\bmod p)$. By Lemma 2.1, we have

$$
q a\left(x^{m}, x^{n}\right)=\left(p^{k}-p^{i}-n, n\right) a\left(x^{p i}, x^{p k-p^{i}}\right) .
$$

Thus if $a\left(x^{m}, x^{n}\right) \neq 0$ then $a\left(x^{p^{i}}, x^{p^{k-p i}}\right) \neq 0$. By anticommutativity of $\Delta$, then $a\left(x^{p k-p^{i}}, x^{p^{i}}\right) \neq 0$. Since the term $x^{m} \otimes x^{n}$ was chosen so that $m d$ was maximum, it follows that

$$
m d \geqq\left(p^{k}-p^{i}\right) d \geqq\left(p^{k}-p^{k-1}\right) d .
$$

Combining this with the inequalities

$$
d p^{k}>d_{1}>m d
$$

and multiplying through by $(p-1) / d p^{k}$ gives

$$
(p-1)>\frac{d_{1}(p-1)}{d p^{k}}>\left(1-\frac{1}{p}\right)(p-1) .
$$

Thus $d_{1}(p-1) / d p^{k}$ is not an integer.

\section{BIBLIOGRAPHY}

1. A. Borel, Sur la cohomologie des espaces fibrés principaux et des espaces homogènes de groupes de Lie compacts, Ann. of Math. vol. 57 (1953). 
2. H. Cartan, Séminaire de Topologie de l'E.N.S., Paris, 1954-1955 (Notes polycopiées).

3. E. Halpern, On the structure of hyperalgebras. Class 1 Hopf algebras, Portugal. Math. vol. 17 fasc. 6 (1959).

4. J. Leray, Sur la forme des espaces topologiques et sur les points fixes des representations, J. Math. Pures Appl. vol. 54 (1945).

5. J. C. Moore, Mimeographed notes of the Algebraic Topology Seminar, Princeton, 1957.

6. H. Samelson, Beiträge zur Topologie der Gruppen-Mannigfaltigkeiten, Ann. of Math. vol. 42 (1941).

7. N. E. Steenrod, Cyclic reduced powers of cohomology classes, Proc. Nat. Acad. Sci. U.S.A. vol. 39 (1953).

UNIVERSITY OF MichigAN

\section{SOME GLOBAL PROPERTIES OF HYPERSURFACES ${ }^{1}$}

ROBERT E. STONG

1. Introduction. The translation theorem of Hopf [1] has been extended by Hsiung [2] and Voss [4] independently to hypersurfaces and by Hsü [3] to other elementary transformations. The purpose of this paper is to extend to hypersurfaces in $(n+1)$-dimensional Euclidean space some results obtained by Hsü [3] for the case $n=2$.

All hypersurfaces mentioned will be assumed to be twice differentiably imbedded in an $(n+1)$-dimensional Euclidean space $E^{n+1}(n+1 \geqq 3)$. The notation used will be that of Hsiung [2]. In particular, $X, N, M_{1}, A$ denote the position vector, unit inner normal, first mean curvature, and area for the hypersurface $V^{n}$. Corresponding quantities for other hypersurfaces will be denoted by ${ }^{*}$, or by primes.

Considerable use will be made of the vector product defined by Hsiung [2]. Namely, if $i_{1}, \cdots, i_{n+1}$ denotes a fixed frame of mutually orthogonal unit vectors and $A_{1}, \cdots, A_{n}$ are $n$ vectors whose components in this frame are $A_{i}^{\alpha}(i=1, \cdots, n ; \alpha=1, \cdots, n+1)$, the vector product is defined by

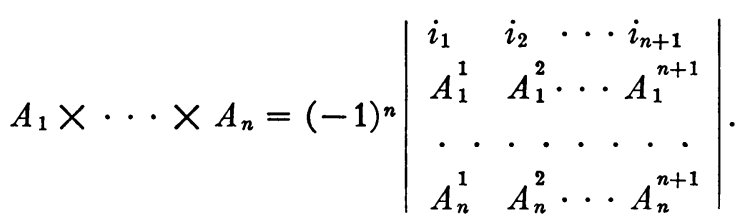

Presented to the Society, June 20, 1959; received by the editors April 20, 1959.

1 This is a portion of a master's thesis at the University of Oklahoma directed by Professor T. K. Pan. 